\title{
On the basic properties of generalized relative order of entire functions
}

\author{
Sanjib Kumar Datta ${ }^{1}$, Tanmay Biswas ${ }^{2}$, Jinarul Haque Shaikh ${ }^{3}$ \\ ${ }^{1}$ Department of Mathematics, University of Kalyani, P.O.-Kalyani, Dist-Nadia, PIN- 741235, West Bengal, India \\ ${ }^{2}$ Rajbari, Rabindrapalli, R. N. Tagore Road, P.O.-Krishnagar, Dist-Nadia, PIN-741101, West Bengal, India \\ ${ }^{3}$ Department of Mathematics, University of Kalyani, P.O.-Kalyani, Dist-Nadia, PIN- 741235, West Bengal, India
}

Received: 18 June 2016, Accepted: 27 February 2017

Published online: 16 August 2017.

\begin{abstract}
Some basic properties in connection to generalized relative order and generalized relative lower order of entire functions have been discussed in this paper.
\end{abstract}

Keywords: Entire functions, generalized relative order, generalized relative lower order, regular relative growth, Property(A).

\section{Introduction, definitions and notations}

Let $\mathbb{C}$ be the set of all finite complex numbers. Also let $f$ be an entire function defined in the open complex plane $\mathbb{C}$. The Nevanlinna's Characteristic function $T_{f}(r)$ and the maximum modulus function $M_{f}(r)$ of $f=\sum_{n=0}^{\infty} a_{n} z^{n}$ on $|z|=r$ are defined as $T_{f}(r)=\frac{1}{2 \pi} \int_{0}^{2 \pi} \log ^{+}\left|f\left(r e^{i \theta}\right)\right| d \theta$ and $M_{f}(r)=\max _{|z|=r}|f(z)|$ respectively where $\log ^{+} x=\max (\log x, 0)$ for all $x \geqslant 0$. Further if $f$ is non-constant then $M_{f}(r)$ is strictly increasing and continuous and its inverse $M_{f}^{-1}:(|f(0)|, \infty) \rightarrow(0, \infty)$ exists and is such that $\lim _{s \rightarrow \infty} M_{f}^{-1}(s)=\infty$. In this connection the following definition is relevant.

Definition 1. [2] A non-constant entire function $f$ is said have the Property (A) if for any $\sigma>1$ and for all large $r$, $\left[M_{f}(r)\right]^{2} \leq M_{f}\left(r^{\sigma}\right)$ holds. For exapmles of functions with or without the Property (A), one may see [2].

For given two entire functions $f$ and $g$, the ratio $\frac{M_{f}(r)}{M_{g}(r)}$ as $r \rightarrow \infty$ is called the growth of $f$ with respect to $g$ in terms of their maximum moduli. For any integer $l \geq 2$, Sato [6] introduced the definitions of generalized order $\rho_{f}^{[l]}$ and generalized lower order $\lambda_{f}^{[l]}$ of an entire function $f$ which are generally used in computational purpose and defined in terms of the growth of $f$ with respect to the exp function of first order in the following way.

$$
\rho_{f}^{[l]}=\limsup _{r \rightarrow \infty} \frac{\log ^{[l]} M_{f}(r)}{\log ^{[2]} M_{\exp z}(r)}=\limsup _{r \rightarrow \infty} \frac{\log ^{[l]} M_{f}(r)}{\log r}
$$

and

$$
\lambda_{f}^{[l]}=\liminf _{r \rightarrow \infty} \frac{\log ^{[l]} M_{f}(r)}{\log ^{[2]} M_{\exp z}(r)}=\liminf _{r \rightarrow \infty} \frac{\log ^{[l]} M_{f}(r)}{\log r}
$$

where $\log ^{[l]} x=\log \left(\log ^{[l-1]} x\right), l=1,2,3, \ldots$ and $\log ^{[0]} x=x$. An entire function $f$ is said to be of regular generalized growth if its generalized order coincides with its generalized lower order; otherwise $f$ is said to be irregular generalized 
growth.

When $l=2$, the above definition coincides with the classical definitions of order and lower order which are as follows:

$$
\rho_{f}=\limsup _{r \rightarrow \infty} \frac{\log \log M_{f}(r)}{\log \log M_{\exp z}(r)}=\limsup _{r \rightarrow \infty} \frac{\log \log M_{f}(r)}{\log r}
$$

and

$$
\lambda_{f}=\liminf _{r \rightarrow \infty} \frac{\log \log M_{f}(r)}{\log \log M_{\exp z}(r)}=\liminf _{r \rightarrow \infty} \frac{\log \log M_{f}(r)}{\log r} .
$$

L. Bernal $\{[1],[2]\}$ introduced the definition of relative order of an entire function $g$ with respect to an entire function $f$ denoted by $\rho_{f}(g)$ to avoid comparing growth just with $\exp z$ in the following way:

$$
\rho_{f}(g)=\inf \left\{\mu>0: M_{g}(r)<M_{f}\left(r^{\mu}\right) \text { for all } r>r_{0}(\mu)>0\right\}=\limsup _{r \rightarrow \infty} \frac{\log M_{f}^{-1} M_{g}(r)}{\log r} .
$$

The above definition coincides with the classical one [7] if $f(z)=\exp z$.

Similarly, one can define the relative lower order of $g$ with respect to $f$, denoted by $\lambda_{f}(g)$ as follows.

$$
\lambda_{f}(g)=\liminf _{r \rightarrow \infty} \frac{\log M_{f}^{-1} M_{g}(r)}{\log r} .
$$

Also an entire function $g$ is said to be of regular relative growth with respect to $f$ if its relative order with respect to $f$ coincides with its relative lower order with respect to $f$.

Extending this notion, Lahiri and Banerjee [5] gave a more generalized concept of relative order which may be given in the following way.

Definition 2. [5] If $l \geq 1$ is a positive integer, then the l-th generalized relative order of $f$ with respect to $g$, denoted by $\rho_{f}^{[l]}(g)$ is defined by

$$
\rho_{g}^{[l]}(f)=\inf \left\{\mu>0: M_{f}(r)<M_{g}\left(\exp ^{[l-1]} r^{\mu}\right) \text { for all } r>r_{0}(\mu)>0 .\right\}=\limsup _{r \rightarrow \infty} \frac{\log { }^{[l]} M_{g}^{-1} M_{f}(r)}{\log r} .
$$

If $l=1$ then $\rho_{g}^{l}(f)=\rho_{g}(f)$. If $l=1, g(z)=\exp z$ then $\rho_{g}^{[l]}(f)=\rho_{f}$, the classical order of $f\{c f .[7]\}$.

Likewise, one can define the l-th generalized relative lower order of $g$ with respect to $f$, denoted by $\lambda_{f}^{[l]}(g)$ as follows :

$$
\lambda_{f}^{[l]}(g)=\liminf _{r \rightarrow \infty} \frac{\log [l] M_{f}^{-1} M_{g}(r)}{\log r}
$$

and also in this case if $\rho_{g}^{[l]}(f)=\lambda_{g}^{[l]}(f)$ then $g$ is said to be of regular l-th generalized relative growth with respect to $f$; otherwise $g$ is said to be of irregular $l$-th generalized relative growth with respect to $f$.

It is well known that the order of products and sums of two entire functions are not greater than the maximal order of the two functions and Bernal [2] extended these results for relative order. Extending this notion, Lahiri and Banerjee [5] established the following two theorems: 
Theorem A. [5] Let $f_{1}, g_{1}$ and $g_{2}$ are any three entire functions. Then

$$
\rho_{f_{1}}^{[l]}\left(g_{1} \pm g_{2}\right) \leq \rho_{f_{1}}^{[l]}\left(g_{i}\right)
$$

where $\rho_{f_{1}}^{[l]}\left(g_{i}\right)=\max \left\{\rho_{f_{1}}^{[l]}\left(g_{k}\right) \mid k=i=1,2\right\}$ and $l \geq 1$. The sign of equality holds when $\rho_{f_{1}}^{[l]}\left(g_{1}\right) \neq \rho_{f_{1}}^{[l]}\left(g_{2}\right)$.

Theorem B. [5] Let $f_{1}, g_{1}$ and $g_{2}$ are any three entire functions. Then

$$
\rho_{f_{1}}^{[l]}\left(g_{1} \cdot g_{2}\right) \leq \rho_{f_{1}}^{[l]}\left(g_{i}\right)
$$

where $\rho_{f_{1}}^{[l]}\left(g_{i}\right)=\max \left\{\rho_{f_{1}}^{[l]}\left(g_{k}\right) \mid k=i=1,2\right\}$ and $l \geq 1$. The sign of equality holds when $\rho_{f_{1}}^{[l]}\left(g_{1}\right) \neq \rho_{f_{1}}^{[l]}\left(g_{2}\right)$. Similar results hold for the quotient $\frac{g_{1}}{g_{2}}$ provided $\frac{g_{1}}{g_{2}}$ is entire.

Datta, Biswas and Biswas [3] also investigated some parallel basic properties of generalized relative lower order of entire functions which are as follows:

Theorem C. [3] Let $f_{1}, f_{2}$ and $g_{1}$ are any three entire functions. Then

$$
\lambda_{f_{1} \pm f_{2}}^{[l]}\left(g_{1}\right) \geq \lambda_{f_{i}}^{[l]}\left(g_{1}\right)
$$

where $\lambda_{f_{i}}^{[l]}\left(g_{1}\right)=\min \left\{\lambda_{f_{k}}^{[l]}\left(g_{1}\right) \mid k=i=1,2\right\}$ and $l \geq 1$. The sign of equality holds when $\lambda_{f_{1}}^{[l]}\left(g_{1}\right) \neq \lambda_{f_{2}}^{[l]}\left(g_{1}\right)$.

Theorem D. [3] Let $f_{1}, f_{2}$ and $g_{1}$ are any three entire functions. Then

$$
\lambda_{f_{1} \cdot f_{2}}^{[l]}\left(g_{1}\right) \geq \lambda_{f_{i}}^{[l]}\left(g_{1}\right)
$$

where $\lambda_{f_{i}}^{[l]}\left(g_{1}\right)=\min \left\{\lambda_{f_{k}}^{[l]}\left(g_{1}\right) \mid k=i=1,2\right\}$ and $l \geq 1$. The sign of equality holds when $\lambda_{f_{1}}^{[l]}\left(g_{1}\right) \neq \lambda_{f_{2}}^{[l]}\left(g_{1}\right)$. Similar results hold for the quotient $\frac{f_{1}}{f_{2}}$ provided $\frac{f_{1}}{f_{2}}$ is entire.

In fact in the present paper, we wish to investigate a few properties of generalized relative order and generalized relative lower order of entire functions under some what different conditions. We do not explain the standard definitions and notations in the theory of entire functions as those are available in [8].

\section{Lemmas}

In this section we present some lemmas which will be needed in the sequel.

Lemma 1. [2] Suppose $f$ is an entire function and $\alpha, \beta$ are such that $\alpha>1$ and $0<\beta<\alpha$. Then

$$
M_{f}(\alpha r)>\beta M_{f}(r) .
$$

Lemma 2.[2] Let $f$ be an entire function which satisfies the Property (A) then for any positive integer $n$, and for all large $r$

$$
\left[M_{f}(r)\right]^{n} \leq M_{f}\left(r^{\delta}\right)
$$

holds where $\delta>1$.

Lemma 3. [5] Every entire function $f$ satisfying the Property $(A)$ is transcendental. 
Lemma 4. [4] Let $f$ be an entire function. Then for all sufficiently large values of $r$,

$$
T_{f}(r) \leq \log M_{f}(r) \leq 3 T_{f}(2 r)\{c f .[4], p .18\}
$$

\section{Theorems}

In this section we present the main results of the paper.

Theorem 1. Let $f_{1}, f_{2}, g_{1}$ and $g_{2}$ be any four entire functions. Then

(i)

$$
\rho_{f_{1} \pm f_{2}}^{[l]}\left(g_{1}\right) \geq \rho_{f_{i}}^{[l]}\left(g_{1}\right)
$$

where $l \geq 1, \rho_{f_{i}}^{[l]}\left(g_{1}\right)=\min \left\{\rho_{f_{k}}^{[l]}\left(g_{1}\right) \mid k=i=1,2\right\}$ and $g_{1}$ is of regular generalized relative growth with respect to at least any one of $f_{1}$ or $f_{2}$. The sign of equality holds when $\rho_{f_{1}}^{[l]}\left(g_{1}\right) \neq \rho_{f_{2}}^{[l]}\left(g_{1}\right)$; and

(ii)

$$
\lambda_{f_{1}}^{[l]}\left(g_{1} \pm g_{2}\right) \leq \lambda_{f_{1}}^{[l]}\left(g_{i}\right)
$$

where $l \geq 1, \lambda_{f_{1}}^{[l]}\left(g_{i}\right)=\max \left\{\lambda_{f_{1}}^{[l]}\left(g_{k}\right) \mid k=i=1,2\right\}$ and at least $g_{1}$ or $g_{2}$ is of regular generalized relative growth with respect to $f_{1}$. The sign of equality holds when $\lambda_{f_{1}}^{[l]}\left(g_{1}\right) \neq \lambda_{f_{1}}^{[l]}\left(g_{2}\right)$.

Proof. From the definition of generalized relative order and generalized relative lower order of entire function, we have for all sufficiently large values of $r$ that

$$
\begin{gathered}
M_{g_{k}}(r) \leq M_{f_{k}}\left[\exp ^{[l-1]}\left(r^{\left(\rho_{f_{k}}^{[l]}\left(g_{k}\right)+\varepsilon\right)}\right)\right], \\
M_{g_{k}}(r) \geq M_{f_{k}}\left[\exp ^{[l-1]} r^{\left(\lambda_{f_{k}}^{[l]}\left(g_{k}\right)-\varepsilon\right)}\right], \text { then } M_{f_{k}}(r) \leq M_{g_{k}}\left[\left(\log \left[{ }^{[l-1]} r\right)^{\frac{1}{\lambda_{f_{k}}^{l l g}\left(g_{k}\right)-\varepsilon}}\right],\right.
\end{gathered}
$$

and also for a sequence values of $r$ tending to infinity we get that

$$
\begin{gathered}
M_{g_{k}}(r) \geq M_{f_{k}}\left[\exp ^{[l-1]}\left(r^{\left(\rho_{f_{k}}^{[l]}\left(g_{k}\right)-\varepsilon\right)}\right)\right], \text { then } M_{f_{k}}(r) \leq M_{g_{k}}\left[(\log [l-1] r)^{\frac{1}{\rho_{f_{k}}^{[l]}\left(g_{k}\right)-\varepsilon}}\right] \\
M_{g_{k}}(r) \leq M_{f_{k}}\left[\exp ^{[l-1]}\left(r^{\left(\lambda_{f_{k}}^{[l]}\left(g_{k}\right)+\varepsilon\right)}\right)\right]
\end{gathered}
$$

where $\varepsilon(>0)$ is any arbitrary positive number and $i=1,2$.

Case I. If $\rho_{f_{1} \pm f_{2}}^{[l]}\left(g_{1}\right)=\infty$ then $\rho_{f_{1} \pm f_{2}}^{[l]}\left(g_{1}\right) \geq \rho_{f_{i}}^{[l]}\left(g_{1}\right)$ is obvious. So we suppose that $\rho_{f_{1} \pm f_{2}}^{[l]}\left(g_{1}\right)<\infty$. We can clearly assume that $\rho_{f_{i}}^{[l]}\left(g_{1}\right) \mid i=1,2$ is finite. Also suppose that $\rho_{f_{i}}^{[l]}\left(g_{1}\right) \leq \rho_{f_{k}}^{[l]}\left(g_{1}\right)$ where $k=i=1,2$ with $f_{i} \neq f_{k}$ and $g_{1}$ is of regular generalized relative growth with respect to at least any one of $f_{1}$ or $f_{2}$. Now in view of Lemma 1 and by (2) and 
(3), we obtain for a sequence of values of $r$ tending to infinity that

$$
\begin{aligned}
& M_{f_{1} \pm f_{2}}(r)<M_{f_{1}}(r)+M_{f_{2}}(r), \text { that is } \\
& M_{f_{1} \pm f_{2}}(r)<\sum_{k=1}^{k=2} M_{g_{1}}\left[\left(\log ^{[l-1]} r\right) \frac{1}{\left(\rho_{f_{k}}^{[l]}\left(g_{1}\right)-\varepsilon\right)}\right], \\
& M_{f_{1} \pm f_{2}}(r)<2 M_{g_{1}}\left[\left(\log ^{[l-1]} r\right) \frac{1}{\left(\rho_{f_{i}}^{[l]}\left(g_{1}\right)-\varepsilon\right)}\right], \\
& M_{f_{1} \pm f_{2}}(r)<M_{g_{1}}\left[3\left(\log ^{[l-1]} r\right) \frac{1}{\left(\rho_{f_{i}}^{[l]}\left(g_{1}\right)-\varepsilon\right)}\right], \\
& M_{f_{1} \pm f_{2}}\left[\exp ^{[l-1]}\left(\left(\frac{r}{3}\right)^{\left(\rho_{f_{i}}^{[l]}\left(g_{1}\right)-\varepsilon\right)}\right)\right]<M_{g_{1}}(r), \\
& M_{f_{1} \pm f_{2}}\left[\exp ^{[l-1]}\left(\left(\frac{r}{3}\right)^{\left(\rho_{f_{i}}^{[l]}\left(g_{1}\right)-\varepsilon\right)}\right)\right]<M_{g_{1}}(r), \\
& \log ^{[l]} \exp ^{[l-1]}\left(\left(\frac{r}{3}\right)\left(\rho_{f_{i}}^{[l]}\left(g_{1}\right)-\varepsilon\right)\right. \\
& \left(\rho_{f_{i}}^{[l]}\left(g_{1}\right)-\varepsilon\right) \log r+O(1)<\log { }^{[l]} M_{f_{1} \pm f_{2}}^{-1} M_{g_{1}}(r), \\
& \left(\rho_{f_{i}}^{[l]}\left(g_{1}\right)-\varepsilon\right)+\frac{O(1)}{\log r}<\frac{\log { }_{f_{1} \pm f_{2}} M_{g_{1}}(r) \text { and } M_{f_{1} \pm f_{2}}^{-1} M_{g_{1}}(r)}{\log r} .
\end{aligned}
$$

Since $\varepsilon>0$ is arbitrary, we get from above that

$$
\rho_{f_{1} \pm f_{2}}^{[l]}\left(g_{1}\right)=\limsup _{r \rightarrow \infty} \frac{\log ^{[l]} M_{f_{1} \pm f_{2}}^{-1} M_{g_{1}}(r)}{\log r} \geq \rho_{f_{i}}^{[l]}\left(g_{1}\right) .
$$

Now without loss of genetality, we may consider that $\rho_{f_{1}}^{[l]}\left(g_{1}\right)<\rho_{f_{2}}^{[l]}\left(g_{1}\right)$ and $f=f_{1} \pm f_{2}$. Then $\rho_{f}^{[l]}\left(g_{1}\right) \geq \rho_{f_{1}}^{[l]}\left(g_{1}\right)$. Further, $f_{1}=\left(f \pm f_{2}\right)$ and in this case we obtain that $\rho_{f_{1}}^{[l]}\left(g_{1}\right) \geq \min \left\{\rho_{f}^{[l]}\left(g_{1}\right), \rho_{f_{2}}^{[l]}\left(g_{1}\right)\right\}$. As we assume that $\rho_{f_{1}}^{[l]}\left(g_{1}\right)<\rho_{f_{2}}^{[l]}\left(g_{1}\right)$, therefore we have $\rho_{f_{1}}^{[l]}\left(g_{1}\right) \geq \rho_{f}^{[l]}\left(g_{1}\right)$ and hence $\rho_{f}^{[l]}\left(g_{1}\right)=\rho_{f_{1}}^{[l]}\left(g_{1}\right)=\min \left\{\rho_{f_{1}}^{[l]}\left(g_{1}\right), \rho_{f_{2}}^{[l]}\left(g_{1}\right)\right\}$. Therefore, $\rho_{f_{1} \pm f_{2}}^{[l]}\left(g_{1}\right)=\rho_{f_{i}}^{[l]}\left(g_{1}\right) \mid i=1,2$ provided $\rho_{f_{1}}^{[l]}\left(g_{1}\right) \neq \rho_{f_{2}}^{[l]}\left(g_{1}\right)$. Thus the first part of the theorem follows.

Case II. If $\lambda_{f_{1}}^{[l]}\left(g_{1} \pm g_{2}\right)=0$ then $\lambda_{f_{1}}^{[l]}\left(g_{1} \pm g_{2}\right) \leq \lambda_{f_{1}}^{[l]}\left(g_{i}\right)$ is obvious. So we suppose that $\lambda_{f_{1}}^{[l]}\left(g_{1} \pm g_{2}\right)>0$. We can clearly assume that $\lambda_{f_{1}}^{[l]}\left(g_{i}\right) \mid i=1,2$ is finite. Also suppose that $\lambda_{f_{1}}^{[l]}\left(g_{k}\right) \leq \lambda_{f_{1}}^{[l]}\left(g_{i}\right)$ where $k=i=1,2$ with $g_{k} \neq g_{i}$ and at least $g_{1}$ or $g_{2}$ is of regular generalized relative growth with respect to $f_{1}$. Now in view of Lemma 1 and using (1) and (4), we get for a sequence of values of $r$ tending to infinity that

$$
\begin{aligned}
& M_{g_{1} \pm g_{2}}(r)<M_{g_{1}}(r)+M_{g_{2}}(r) \text { that is } \\
& M_{g_{1} \pm g_{2}}(r)<\sum_{k=1}^{k=2} M_{f_{1}}\left[\exp ^{[l-1]}\left(r^{\left(\lambda_{f_{1}}^{[l]}\left(g_{k}\right)+\varepsilon\right)}\right)\right] \\
& M_{g_{1} \pm g_{2}}(r)<2 M_{f_{1}}\left[\exp ^{[l-1]}\left(r^{\left(\lambda_{f_{1}}^{[l]}\left(g_{i}\right)+\varepsilon\right)}\right)\right]
\end{aligned}
$$




$$
\begin{aligned}
& M_{g_{1} \pm g_{2}}(r)<M_{f_{1}}\left[3 \exp ^{[l-1]}\left(r^{\left(\lambda_{f_{1}}^{[l]}\left(g_{i}\right)+\varepsilon\right)}\right)\right], \\
& M_{g_{1} \pm g_{2}}(r)<M_{f_{1}}\left[\exp ^{[l-1]}\left(3(l-1) r^{\left(\lambda_{f_{1}}^{[l]}\left(g_{i}\right)+\varepsilon\right)}\right)\right], \\
& \log ^{[l]} M_{f_{1}}^{-1} M_{g_{1} \pm g_{2}}(r)<\left(\lambda_{f_{1}}^{[l]}\left(g_{i}\right)+\varepsilon\right) \log r+O(1) \text { and } \\
& \frac{\log ^{[l]} M_{f_{1}}^{-1} M_{g_{1} \pm g_{2}}(r)}{\log r}<\frac{\left(\lambda_{f_{1}}^{[l]}\left(g_{i}\right)+\varepsilon\right) \log r+O(1)}{\log r} .
\end{aligned}
$$

Since $\varepsilon>0$ is arbitrary, it follows from above that

$$
\text { i.e., } \lambda_{f_{1}}^{[l]}\left(g_{1} \pm g_{2}\right)=\liminf _{r \rightarrow \infty} \frac{\log ^{[l]} M_{f_{1}}^{-1} M_{g_{1} \pm g_{2}}(r)}{\log r} \leq \lambda_{f_{1}}^{[l]}\left(g_{i}\right)
$$

Further without loss of genetality, let $\lambda_{f_{1}}^{[l]}\left(g_{1}\right)<\lambda_{f_{1}}^{[l]}\left(g_{2}\right)$ and $g=g_{1} \pm g_{2}$. Then $\lambda_{f_{1}}^{[l]}(g) \leq \lambda_{f_{1}}^{[l]}\left(g_{2}\right)$. Further, $g_{2}= \pm\left(g-g_{1}\right)$ and in this case we obtain that $\lambda_{f_{1}}^{[l]}\left(g_{2}\right) \leq \max \left\{\lambda_{f_{1}}^{[l]}(g), \lambda_{f_{1}}^{[l]}\left(g_{1}\right)\right\}$. As we assume that $\lambda_{f_{1}}^{[l]}\left(g_{1}\right)<\lambda_{f_{1}}^{[l]}\left(g_{2}\right)$, therefore we have $\lambda_{f_{1}}^{[l]}\left(g_{2}\right) \leq \lambda_{f_{1}}^{[l]}(g)$ and hence $\lambda_{f_{1}}^{[l]}(g)=\lambda_{f_{1}}^{[l]}\left(g_{2}\right)=\max \left\{\lambda_{f_{1}}^{[l]}\left(g_{1}\right), \lambda_{f_{1}}^{[l]}\left(g_{2}\right)\right\}$. Therefore, $\lambda_{f_{1}}^{[l]}\left(g_{1} \pm g_{2}\right)=\lambda_{f_{1}}^{[l]}\left(g_{i}\right) \mid i=$ 1,2 provided $\lambda_{f_{1}}^{[l]}\left(g_{1}\right) \neq \lambda_{f_{1}}^{[l]}\left(g_{2}\right)$. Thus the second part of the theorem is established.

In the line of Theorem A, Theorem C and Theorem 1, one may state the following theorem without its proof.

Theorem 2. Let $f_{1}, f_{2}, g_{1}$ and $g_{2}$ be any four entire functions. Then for $l \geq 1$,

(i)

$$
\rho_{f_{1} \pm f_{2}}^{[l]}\left(g_{1} \pm g_{2}\right) \leq \max \left[\min \left\{\rho_{f_{1}}^{[l]}\left(g_{1}\right), \rho_{f_{2}}^{[l]}\left(g_{1}\right)\right\}, \min \left\{\rho_{f_{1}}^{[l]}\left(g_{2}\right), \rho_{f_{2}}^{[l]}\left(g_{2}\right)\right\}\right]
$$

when $\rho_{f_{1}}^{[l]}\left(g_{1}\right) \neq \rho_{f_{2}}^{[l]}\left(g_{1}\right), \rho_{f_{1}}^{[l]}\left(g_{2}\right) \neq \rho_{f_{2}}^{[l]}\left(g_{2}\right)$ and $g_{1}$ and $g_{1}$ are both of regular generalized relative growth with respect to at least any one of $f_{1}$ or $f_{2}$. The sign of equality holds when (ii) $\min \left\{\rho_{f_{1}}^{[l]}\left(g_{1}\right), \rho_{f_{2}}^{[l]}\left(g_{1}\right)\right\} \neq \min \left\{\rho_{f_{1}}^{[l]}\left(g_{2}\right), \rho_{f_{2}}^{[l]}\left(g_{2}\right)\right\} ;$ and

$$
\lambda_{f_{1} \pm f_{2}}^{[l]}\left(g_{1} \pm g_{2}\right) \geq \min \left[\max \left\{\lambda_{f_{1}}^{[l]}\left(g_{1}\right), \lambda_{f_{2}}^{[l]}\left(g_{1}\right)\right\}, \max \left\{\lambda_{f_{1}}^{[l]}\left(g_{2}\right), \lambda_{f_{2}}^{[l]}\left(g_{2}\right)\right\}\right]
$$

when $\lambda_{f_{1}}^{[l]}\left(g_{1}\right) \neq \lambda_{f_{2}}^{[l]}\left(g_{1}\right), \lambda_{f_{1}}^{[l]}\left(g_{2}\right) \neq \lambda_{f_{2}}^{[l]}\left(g_{2}\right)$ and at least $g_{1}$ or $g_{2}$ is of regular generalized relative growth with respect to $f_{1}$ and $f_{2}$ respectively. The sign of equality holds when $\max \left\{\lambda_{f_{1}}^{[l]}\left(g_{1}\right), \lambda_{f_{2}}^{[l]}\left(g_{1}\right)\right\} \neq \max \left\{\lambda_{f_{1}}^{[l]}\left(g_{2}\right), \lambda_{f_{2}}^{[l]}\left(g_{2}\right)\right\}$.

Theorem 3. Let $f_{1}, f_{2}, g_{1}$ and $g_{2}$ be any four entire functions. Then

(i)

$$
\rho_{f_{1} \cdot f_{2}}^{[l]}\left(g_{1}\right) \geq \rho_{f_{i}}^{[l]}\left(g_{1}\right)
$$

where $l \geq 1, \rho_{f_{i}}^{[l]}\left(g_{1}\right)=\min \left\{\rho_{f_{k}}^{[l]}\left(g_{1}\right) \mid k=i=1,2\right\}, g_{1}$ has the Property $(A)$ and also $g_{1}$ is of regular generalized relative growth with respect to at least any one of $f_{1}$ or $f_{2}$. The sign of equality holds when $\rho_{f_{1}}^{[l]}\left(g_{1}\right) \neq \rho_{f_{2}}^{[l]}\left(g_{1}\right)$. (ii) Similar results hold for the quotient $\frac{f_{1}}{f_{2}}$ provided $\frac{f_{1}}{f_{2}}$ is entire, and

$$
\lambda_{f_{1}}^{[l]}\left(g_{1} \cdot g_{2}\right) \leq \lambda_{f_{1}}^{[l]}\left(g_{i}\right)
$$

where $l \geq 1, \lambda_{f_{1}}^{[l]}\left(g_{i}\right)=\max \left\{\lambda_{f_{1}}^{[l]}\left(g_{k}\right) \mid k=i=1,2\right\}, f_{1}$ has the Property (A) and at least $g_{1}$ or $g_{2}$ is of regular generalized relative growth with respect to $f_{1}$. The sign of equality holds when $\lambda_{f_{1}}^{[l]}\left(g_{1}\right) \neq \lambda_{f_{1}}^{[l]}\left(g_{2}\right)$. Similar results hold for the quotient $\frac{g_{1}}{g_{2}}$ provided $\frac{g_{1}}{g_{2}}$ is entire. 
Proof. For any two entire functions $h_{1}$ and $h_{2}$, we have for all sufficiently large values of $r$ that

$$
T_{h_{1} \cdot h_{2}}(r) \leq T_{h_{1}}(r)+T_{h_{2}}(r) .
$$

Case I. By Lemma $3, g_{1}$ is transcendental. Suppose that $\rho_{f_{1} \cdot f_{2}}^{[l]}\left(g_{1}\right)<\infty$. Otherwise if $\rho_{f_{1} \cdot f_{2}}^{[l]}\left(g_{1}\right)=\infty$ then the result is obvious. We can clearly assume that $\rho_{f_{i}}^{[l]}\left(g_{1}\right) \mid i=1,2$ is finite. Also suppose that $\rho_{f_{i}}^{[l]}\left(g_{1}\right) \leq \rho_{f_{k}}^{[l]}\left(g_{1}\right)$ where $k=i=1,2$ with $f_{i} \neq f_{k}$ and $g_{1}$ is of regular generalized relative growth with respect to at least any one of $f_{1}$ or $f_{2}$,. Now in view of Lemma 2, Lemma 4 and also by (2) and (3) we get from (5) (considering $h=f$ in (5)) for a sequence of values of $r$ tending to infinity that

$$
\begin{aligned}
& \frac{1}{3} \log M_{f_{1} \cdot f_{2}}\left(\frac{r}{2}\right) \leq \log M_{f_{1}}(r)+\log M_{f_{2}}(r) \text { that is } \\
& \frac{1}{3} \log M_{f_{1} \cdot f_{2}}\left(\frac{r}{2}\right) \leq \sum_{k=1}^{k=2} \log M_{g_{1}}\left[\left(\log ^{[l-1]} r\right)^{\frac{1}{\rho_{f_{k}} \frac{1}{\left(g_{1}\right)-\varepsilon}}}\right] \text {, } \\
& \frac{1}{3} \log M_{f_{1} \cdot f_{2}}\left(\frac{r}{2}\right) \leq 2 \log M_{g_{1}}\left[\left(\log { }^{[l-1]} r\right)^{\frac{1}{\rho_{f_{k}}^{[l]}\left(g_{1}\right)-\varepsilon}}\right], \\
& \log M_{f_{1} \cdot f_{2}}\left(\frac{r}{2}\right) \leq 6 \log M_{g_{1}}\left[\left(\log \left[{ }^{[l-1]} r\right)^{\frac{1}{\rho_{f_{i}}^{l l}\left(g_{1}\right)-\varepsilon}}\right],\right. \\
& M_{f_{1} \cdot f_{2}}\left(\frac{r}{2}\right) \leq\left[M_{g_{1}}\left[\left(\log ^{[l-1]} r\right)^{\frac{1}{\rho_{f_{i}}\left(g_{1}\right)-\varepsilon}}\right]\right]^{6}, \\
& M_{f_{1} \cdot f_{2}}\left(\frac{r}{2}\right) \leq M_{g_{1}}\left[\left(\log ^{[l-1]} r\right)^{\frac{\delta}{\rho_{f_{i}}^{[l]}\left(g_{1}\right)-\varepsilon}}\right], \\
& M_{f_{1} \cdot f_{2}}\left(\frac{\exp ^{[l-1]} r^{\frac{\rho_{f_{i}}^{[l]}\left(g_{1}\right)-\varepsilon}{\delta}}}{2}\right) \leq M_{g_{1}}(r) \\
& \log \left[\frac{\exp ^{[l-1]} r^{\frac{\rho_{f_{i}}^{[l]}\left(g_{1}\right)-\varepsilon}{\delta}}}{2}\right) \leq \log ^{[l]} M_{f_{1} \cdot f_{2}}^{-1} M_{g_{1}}(r), \\
& \left(\frac{\rho_{f_{i}}^{[l]}\left(g_{1}\right)-\varepsilon}{\sigma}\right) \log r+O(1) \leq \log ^{[l]} M_{f_{1} \cdot f_{2}}^{-1} M_{g_{1}}(r) \text { and } \\
& \frac{\rho_{f_{i}}^{[l]}\left(g_{1}\right)}{\sigma}-\frac{\varepsilon}{\sigma}+\frac{O(1)}{\log r} \leq \frac{\log ^{[l]} M_{f_{1} \cdot f_{2}}^{-1} M_{g_{1}}(r)}{\log r} .
\end{aligned}
$$

Since $\varepsilon>0$ is arbitrary, we obtain by letting $\sigma \rightarrow 1+$,

$$
\rho_{f_{1} \cdot f_{2}}^{[l]}\left(g_{1}\right)=\limsup _{r \rightarrow \infty} \frac{\log ^{[l]} M_{f_{1} \cdot f_{2}}^{-1} M_{g_{1}}(r)}{\log r} \geq \rho_{f_{i}}^{[l]}\left(g_{1}\right) \text {. }
$$

Now without loss of genetality, we may consider that $\rho_{f_{1}}^{[l]}\left(g_{1}\right)<\rho_{f_{2}}^{[l]}\left(g_{1}\right)$ and $f=f_{1} \cdot f_{2}$. Then $\rho_{f}^{[l]}\left(g_{1}\right) \geq \rho_{f_{1}}^{[l]}\left(g_{1}\right)$. Further, $f_{1}=\frac{f}{f_{2}}$ and and $T_{f_{2}}(r)=T_{\frac{1}{f_{2}}}(r)+O(1)$. Therefore $T_{f_{1}}(r) \leq T_{f}(r)+T_{f_{2}}(r)+O(1)$, and in this case we obtain 
that $\rho_{f_{1}}^{[l]}\left(g_{1}\right) \geq \min \left\{\rho_{f}^{[l]}\left(g_{1}\right), \rho_{f_{2}}^{[l]}\left(g_{1}\right)\right\}$. As we assume that $\rho_{f_{1}}^{[l]}\left(g_{1}\right)<\rho_{f_{2}}^{[l]}\left(g_{1}\right)$, so we have $\rho_{f_{1}}^{[l]}\left(g_{1}\right) \geq \rho_{f}^{[l]}\left(g_{1}\right)$ and hence $\rho_{f}^{[l]}\left(g_{1}\right)=\rho_{f_{1}}^{[l]}\left(g_{1}\right)=\min \left\{\rho_{f_{1}}^{[l]}\left(g_{1}\right), \rho_{f_{2}}^{[l]}\left(g_{1}\right)\right\}$. Therefore, $\rho_{f_{1} \cdot f_{2}}^{[l]}\left(g_{1}\right)=\rho_{f_{i}}^{[l]}\left(g_{1}\right) \mid i=1,2$ provided $\rho_{f_{1}}^{[l]}\left(g_{1}\right) \neq \rho_{f_{2}}^{[l]}\left(g_{1}\right)$.

Further suppose that $f=\frac{f_{1}}{f_{2}}$ with $f_{1}, f_{2}, f$ entire and let $\rho_{f_{1}}^{[l]}\left(g_{1}\right) \geq \rho_{f_{2}}^{[l]}\left(g_{1}\right)$. We have $f_{1}=f \cdot f_{2}$. Therefore $\rho_{f_{1}}^{[l]}\left(g_{1}\right)=\rho_{f}^{[l]}\left(g_{1}\right)$ if $\rho_{f}^{[l]}\left(g_{1}\right)<\rho_{f_{2}}^{[l]}\left(g_{1}\right)$. So it follows that $\rho_{f_{1}}^{[l]}\left(g_{1}\right)<\rho_{f_{2}}^{[l]}\left(g_{1}\right)$, which contradicts the hypothesis $\rho_{f_{1}}^{[l]}\left(g_{1}\right) \geq \rho_{f_{2}}^{[l]}\left(g_{1}\right)$ ". Hence $\rho_{f}^{[l]}\left(g_{1}\right)=\rho_{\frac{f_{1}}{f_{2}}}^{[l]}\left(g_{1}\right) \geq \rho_{f_{2}}^{[l]}\left(g_{1}\right)=\min \left\{\rho_{f_{1}}^{[l]}\left(g_{1}\right), \rho_{f_{2}}^{[l]}\left(g_{1}\right)\right\}$. Also suppose that $\rho_{f_{1}}^{[l]}\left(g_{1}\right)>\rho_{f_{2}}^{[l]}\left(g_{1}\right)$. Then $\rho_{f_{1}}^{[l]}\left(g_{1}\right)=\min \left\{\rho_{f}^{[l]}\left(g_{1}\right), \rho_{f_{2}}^{[l]}\left(g_{1}\right)\right\}=\rho_{f_{2}}^{[l]}\left(g_{1}\right)$, if $\rho_{f}^{[l]}\left(g_{1}\right)>\rho_{f_{2}}^{[l]}\left(g_{1}\right)$, which is also a contradiction. Thus $\rho_{f}^{[l]}\left(g_{1}\right)=\rho_{\frac{f_{1}}{f_{2}}}^{[l]}\left(g_{1}\right)=\min \left\{\rho_{f_{1}}^{[l]}\left(g_{1}\right), \rho_{f_{2}}^{[l]}\left(g_{1}\right)\right\}$. Thus the first part of the theorem is established.

Case II. By Lemma $3, g_{1}$ is transcendental. If $\lambda_{f_{1}}^{[l]}\left(g_{1} \cdot g_{2}\right)=0$ then $\lambda_{f_{1}}^{[l]}\left(g_{1} \cdot g_{2}\right) \leq \lambda_{f_{1}}^{[l]}\left(g_{i}\right)$ is obvious. So we suppose that $\lambda_{f_{1}}^{[l]}\left(g_{1} \cdot g_{2}\right)>0$. We can clearly assume that $\lambda_{f_{1}}^{[l]}\left(g_{i}\right) \mid i=1,2$ is finite. Also suppose that $\lambda_{f_{1}}^{[l]}\left(g_{k}\right) \leq \lambda_{f_{1}}^{[l]}\left(g_{i}\right)$ where $k=i=1,2$ with $g_{k} \neq g_{i}$ and at least $g_{1}$ or $g_{2}$ is of regular generalized relative growth with respect to $f_{1}$. Now in view of Lemma 2, Lemma 4 and using (1) and (4) we get from (5) (considering $h=g$ in (5)) for a sequence of values of $r$ tending to infinity that

$$
\begin{aligned}
& \frac{1}{3} \log M_{g_{1} \cdot g_{2}}\left(\frac{r}{2}\right) \leq \log M_{g_{1}}(r)+\log M_{g_{2}}(r) \text { that } \\
& \frac{1}{3} \log M_{g_{1} \cdot g_{2}}\left(\frac{r}{2}\right) \leq \sum_{k=1}^{k=2} \log M_{f_{1}}\left[\exp ^{[l-1]}\left(r^{\left(\lambda^{[l]} \rho_{f_{1}}\left(g_{k}\right)+\varepsilon\right)}\right)\right], \\
& \frac{1}{3} \log M_{g_{1} \cdot g_{2}}\left(\frac{r}{2}\right) \leq 2 \log M_{f_{1}}\left[\exp ^{[l-1]}\left(r^{\left(\lambda^{[l]} \rho_{f_{1}}\left(g_{k}\right)+\varepsilon\right)}\right)\right], \\
& \log M_{g_{1} \cdot g_{2}}\left(\frac{r}{2}\right) \leq 6 \log M_{f_{1}}\left[\exp ^{[l-1]}\left(r^{\left(\lambda^{[l]} \rho_{f_{1}}\left(g_{i}\right)+\varepsilon\right)}\right)\right] \\
& M_{g_{1} \cdot g_{2}}\left(\frac{r}{2}\right) \leq M_{f_{1}}\left[\exp ^{[l-1]}\left(r^{\left(\lambda^{[l]} \rho_{f_{1}}\left(g_{i}\right)+\varepsilon\right)}\right)\right]^{6} \\
& M_{g_{1} \cdot g_{2}}\left(\frac{r}{2}\right) \leq M_{f_{1}}\left[\exp ^{[l-1]}\left(r^{\delta\left(\lambda_{f_{1}}^{[l]}\left(g_{i}\right)+\varepsilon\right)}\right)\right] \\
& \log ^{[l]} M_{f_{1}}^{-1} M_{g_{1} \cdot g_{2}}\left(\frac{r}{2}\right) \leq \delta\left(\lambda_{f_{1}}^{[l]}\left(g_{i}\right)+\varepsilon\right) \log r, \text { and } \\
& \frac{\log ^{[l]} M_{f_{1}}^{-1} M_{g_{1} \cdot g_{2}}\left(\frac{r}{2}\right)}{\log \left(\frac{r}{2}\right)} \leq \frac{\delta\left(\lambda_{f_{1}}^{[l]}\left(g_{i}\right)+\varepsilon\right) \log r}{\log r+O(1)} .
\end{aligned}
$$

As $\varepsilon>0$ is arbitrary, we get from above by letting $\sigma \rightarrow 1+$,

$$
\lambda_{f_{1}}^{[l]}\left(g_{1} \cdot g_{2}\right)=\liminf _{r \rightarrow \infty} \frac{\log ^{[l]} M_{f_{1} \cdot f_{2}}^{-1} M_{g_{1}}(r)}{\log r} \leq \lambda_{f_{1}}^{[l]}\left(g_{i}\right) .
$$

Moreover without loss of generality, let $\lambda_{f_{1}}^{[l]}\left(g_{1}\right)<\lambda_{f_{1}}^{[l]}\left(g_{2}\right)$ and $g=g_{1} \cdot g_{2}$. Then $\lambda_{f_{1}}^{[l]}(g) \leq \lambda_{f_{1}}^{[l]}\left(g_{2}\right)$. Further, $g_{2}=\frac{g}{g_{1}}$ and and $T_{g_{1}}(r)=T_{\frac{1}{g_{1}}}(r)+O(1)$. Therefore $T_{g_{2}}(r) \leq T_{g}(r)+T_{g_{1}}(r)+O(1)$, and in this case we obtain that $\lambda_{f_{1}}^{[l]}\left(g_{2}\right) \leq \max \left\{\lambda_{f_{1}}^{[l]}(g), \lambda_{f_{1}}^{[l]}\left(g_{1}\right)\right\}$. As we assume that $\lambda_{f_{1}}^{[l]}\left(g_{1}\right)<\lambda_{f_{1}}^{[l]}\left(g_{2}\right)$, therefore we have $\lambda_{f_{1}}^{[l]}\left(g_{2}\right) \leq \lambda_{f_{1}}^{[l]}(g)$ and hence $\lambda_{f_{1}}^{[l]}(g)=\lambda_{f_{1}}^{[l]}\left(g_{2}\right)=\max \left\{\lambda_{f_{1}}^{[l]}\left(g_{1}\right), \lambda_{f_{1}}^{[l]}\left(g_{2}\right)\right\}$. Therefore, $\lambda_{f_{1}}^{[l]}\left(g_{1} \cdot g_{2}\right)=\lambda_{f_{1}}^{[l]}\left(g_{i}\right) \mid i=1,2$ provided $\lambda_{f_{1}}^{[l]}\left(g_{1}\right) \neq \lambda_{f_{1}}^{[l]}\left(g_{2}\right)$.

Now let $g=\frac{g_{1}}{g_{2}}$ with $g_{1}, g_{2}, g$ entire, and suppose $\lambda_{f_{1}}^{[l]}\left(g_{1}\right) \leq \lambda_{f_{1}}^{[l]}\left(g_{2}\right)$. We have $g_{1}=g \cdot g_{2}$. Therefore $\lambda_{f_{1}}^{[l]}\left(g_{1}\right)=\lambda_{f_{1}}^{[l]}(g)$ 
if $\lambda_{f_{1}}^{[l]}(g)>\lambda_{f_{1}}^{[l]}\left(g_{2}\right)$. So it follows that $\lambda_{f_{1}}^{[l]}\left(g_{1}\right)>\lambda_{f_{1}}^{[l]}\left(g_{2}\right)$, which contradicts the hypothesis “ $\lambda_{f_{1}}^{[l]}\left(g_{1}\right) \leq \lambda_{f_{1}}^{[l]}\left(g_{2}\right)$ ". Hence $\lambda_{f_{1}}^{[l]}(g)=\lambda_{f_{1}}^{[l]}\left(\frac{g_{1}}{g_{2}}\right) \leq \lambda_{f_{1}}^{[l]}\left(g_{2}\right)=\max \left\{\lambda_{f_{1}}^{[l]}\left(g_{1}\right), \lambda^{[l]} f_{1}\left(g_{2}\right)\right\}$. Also suppose that $\lambda_{f_{1}}^{[l]}\left(g_{1}\right)>\lambda_{f_{1}}^{[l]}\left(g_{2}\right)$. Then $\lambda_{f_{1}}^{[l]}\left(g_{1}\right)=\max \left\{\lambda_{f_{1}}^{[l]}(g), \lambda_{f_{1}}^{[l]}\left(g_{2}\right)\right\}=\lambda_{f_{1}}^{[l]}\left(g_{2}\right)$, if $\lambda_{f_{1}}^{[l]}(g)<\lambda_{f_{1}}^{[l]}\left(g_{2}\right)$, which is also a contradiction. Thus $\lambda_{f 1}^{[l]}(g)=\lambda_{f_{1}}^{[l]}\left(\frac{g_{1}}{g_{2}}\right)=\max \left\{\lambda_{f_{1}}^{[l]}\left(g_{1}\right), \lambda^{[l]} \rho_{f_{1}}\left(g_{2}\right)\right\}$. Thus the second part of the theorem follows.

The proof of Theorem 4 is omitted because it can be carried in view of Theorem B, Theorem $\mathrm{D}$ and Therorem 3 .

Theorem 4. Let $f_{1}, f_{2}, g_{1}$ and $g_{2}$ be any four entire functions. Then for $l \geq 1$,

$$
\begin{aligned}
& \text { (a) } \rho_{f_{1} \cdot f_{2}}^{[l]}\left(g_{1} \cdot g_{2}\right) \leq \max \left[\min \left\{\rho_{f_{1}}^{[l]}\left(g_{1}\right), \rho_{f_{2}}^{[l]}\left(g_{1}\right)\right\}, \min \left\{\rho_{f_{1}}^{[l]}\left(g_{2}\right), \rho_{f_{2}}^{[l]}\left(g_{2}\right)\right\}\right], \\
& \text { (b) } \rho_{\frac{f_{1}}{f_{2}}}^{[l]}\left(\frac{g_{1}}{g_{2}}\right) \leq \max \left[\min \left\{\rho_{f_{1}}^{[l]}\left(g_{1}\right), \rho_{f_{2}}^{[l]}\left(g_{1}\right)\right\}, \min \left\{\rho_{f_{1}}^{[l]}\left(g_{2}\right), \rho_{f_{2}}^{[l]}\left(g_{2}\right)\right\}\right]
\end{aligned}
$$

when $(i) \rho_{f_{1}}^{[l]}\left(g_{1}\right) \neq \rho_{f_{2}}^{[l]}\left(g_{1}\right),($ ii $) \rho_{f_{1}}^{[l]}\left(g_{2}\right) \neq \rho_{f_{2}}^{[l]}\left(g_{2}\right)$ (iii) $f_{1} \cdot f_{2}, g_{1}$ and $g_{2}$ have the Property (A) and (iv) $g_{1}$ and $g_{1}$ are both of regular generalized relative growth with respect to at least any one of $f_{1}$ or $f_{2}$. The sign of equality holds when $\min \left\{\rho_{f_{1}}^{[l]}\left(g_{1}\right), \rho_{f_{2}}^{[l]}\left(g_{1}\right)\right\} \neq \min \left\{\rho_{f_{1}}^{[l]}\left(g_{2}\right), \rho_{f_{2}}^{[l]}\left(g_{2}\right)\right\} ;$ and

$$
\begin{aligned}
& \text { (c) } \lambda_{f_{1} \cdot f_{2}}^{[l]}\left(g_{1} \cdot g_{2}\right) \geq \min \left[\max \left\{\lambda_{f_{1}}^{[l]}\left(g_{1}\right), \lambda_{f_{2}}^{[l]}\left(g_{1}\right)\right\}, \max \left\{\lambda_{f_{1}}^{[l]}\left(g_{2}\right), \lambda_{f_{2}}^{[l]}\left(g_{2}\right)\right\}\right], \\
& \text { (d) } \lambda_{\frac{f_{1}}{f_{2}}}^{[l]}\left(\frac{g_{1}}{g_{2}}\right) \geq \min \left[\max \left\{\lambda_{f_{1}}^{[l]}\left(g_{1}\right), \lambda_{f_{2}}^{[l]}\left(g_{1}\right)\right\}, \max \left\{\lambda_{f_{1}}^{[l]}\left(g_{2}\right), \lambda_{f_{2}}^{[l]}\left(g_{2}\right)\right\}\right]
\end{aligned}
$$

when (i) $\lambda_{f_{1}}^{[l]}\left(g_{1}\right) \neq \lambda_{f_{2}}^{[l]}\left(g_{1}\right)$, (ii) $\lambda_{f_{1}}^{[l]}\left(g_{2}\right) \neq \lambda_{f_{2}}^{[l]}\left(g_{2}\right)$, (iii) $g_{1} \cdot g_{2}, f_{1}$ and $f_{2}$ have the Property (A) and (iv) at least $g_{1}$ or $g_{2}$ is of regular generalized relative growth with respect to $f_{1}$ and $f_{2}$ respectively. The sign of equality holds when $\max \left\{\lambda_{f_{1}}^{[l]}\left(g_{1}\right), \lambda_{f_{2}}^{[l]}\left(g_{1}\right)\right\} \neq \max \left\{\lambda_{f_{1}}^{[l]}\left(g_{2}\right), \lambda_{f_{2}}^{[l]}\left(g_{2}\right)\right\}$.

\section{Conclusion}

The relative order of growth gives a quantitative assessment of how different functions scale each other and to what extent they are self-similar in growth. The concepts of relative order between two entire functions was initiated in order to avoid comparing growth just with $\exp z$. In the present paper the concept of relative order has been extended up to generalized relative order in different directions. From this view point, the results as proved in the paper show the novelity of the work carried out here and those must be helpful to the future workers of this branch.

\section{Competing interests}

The authors declare that they have no competing interests.

\section{Authors' contributions}

All authors have contributed to all parts of the article. All authors read and approved the final manuscript. 


\section{References}

[1] L. Bernal : Crecimiento relativo de funciones enteras. Contribución al estudio de lasfunciones enteras coníndice exponencial finito, Doctoral Dissertation, University of Seville, Spain, 1984.

[2] L. Bernal : Orden relative de crecimiento de funciones enteras, Collect. Math., Vol. 39 (1988), pp.209-229.

[3] S. K. Datta, T. Biswas, C. Biswas , : Generalized relative lower order of entire functions, Mathematicki Vesnik, Accepted for publication.

[4] W. K. Hayman : Meromorphic functions, The Clarendon Press, Oxford (1964).

[5] B. K. Lahiri, D. Banerjee : Generalised relative order of entire functions, Proc. Nat. Acad. Sci. India, Vol 72(A), No. IV (2002), pp. 351-371.

[6] D. Sato, : On the rate of growth of entire functions of fast growth, Bull. Amer. Math. Soc., Vol. 69 (1963), pp. 411-414.

[7] E. C. Titchmarsh : The Theory of Functions, 2nd ed. Oxford University Press, Oxford (1968).

[8] G. Valiron : Lectures on the General Theory of Integral Functions, Chelsea Publishing Company (1949). 